\title{
Control Theory and Technology
}

http://link.springer.com/journal/11768

DOI https://doi.org/10.1007/s11768-018-7114-5

\section{Editorial}

\section{A special issue to mark the $\mathbf{1 0}^{\text {th }}$ Anniversary of the Passing of Prof. Jingqing Han, a scholar ahead of his time}

Prof. Jingqing Han left us ten years ago but his ideas not only lived on but also grew evermore powerful, as evident in this special issue at Control Theory and Technology, in English, and in the special issue at Control Theory and Applications, in Chinese.

A man of few words, Han was not a person easily understood, even for those close to him. It sometimes took us years to unpack a single idea of his and unearth a brilliant insight. For the benefits of a broader audience, however, let's start with the two basic questions that drove his research:

1) Is the problem of control a problem of stability and optimization, as commonly framed in applied mathematics?

2) If control design cannot be premised on detailed and accurate mathematical model, as declared by H.S. Tsien (Qian Xuesen) in the groundbreaking book, Engineering Cybernetics, in 1954, then what should or could it be premised on?

Han's answer to the first question was a resounding No and its consequence is profound, for it goes against the reigning paradigm and it implies that the right kind of control theory has yet to come. In conceiving ADRC (active disturbance rejection control), his sole purpose was to show us an alternative to the reigning paradigm. In essence, Han solved a problem of control, the existence of which escaped the attention of most scholars in his time. "He was ahead of his time", said Prof. Lei Guo, a colleague of his for many years.

The second question is no less disrupting and mystifying: disrupting because its answer has changed the way control is practiced, as evident in the "application" papers in this issue; mystifying because of the absence of fully articulated principles and the theoretical foundation. In fact, these two questions arose from the same puzzle left by Han: if there is an alternative to modern control theory, what does it entail?

To demystify is to see the world through Han's eyes. Han was trained as mathematician and, as a young scholar, worked with some of the most brilliant mathematicians in China: Hua Luogeng, Guan Zhaozhi, et al. came to mind. His scholarly work was highly regarded from the early 1960s to the late 1980s in the area of optimal control and linear system theory, among others. It was in 1989, at the peak of his career, when he turned his back on, in his words, "theory of model" and went back to square one, i.e., the two questions above. For the next twenty years, Han cultivated the field of control as an experimental science, outside the axiomatic framework pioneered by Prof. R. E. Kalman in the early 1960s.

In doing so Han demonstrated remarkable intellectual honesty and courage. This was no spat with colleagues or one-upmanship in scholarly debate. This was an awakening to which Han dedicated the rest of his life: he saw what needs to be done and he was willing to sacrifice his career for it. This is because control theory, as Han saw it, is more than a model-based study of stability and optimality. If our research is to be authentic, if our work is to impact

(C) 2018 South China University of Technology, Academy of Mathematics and Systems Science, CAS and Springer-Verlag GmbH Germany, part of Springer Nature 
practice, we must go beyond the framework borrowed from applied mathematics, particularly its tacit premise on mathematical model first exposed by H. S. Tsien in 1954.

Han was not alone, but he was the most profound. Much has been done on the problem of uncertainty and disturbance, respectively in and outside of the reigning paradigm, to make control systems "robust", "adaptive", "intelligent", "model-free", "data-driven", and so on. Han is different in both the depth of his grasp of the root problem and the thoroughness in his solution, Active Disturbance Rejection Control (ADRC). By estimating and canceling in real time the "total disturbance" defined in the context of Han's Canonical (cascade integral) Form, to which a large class of nonlinear plants can be reduced, as shown by Prof. Hebertt Sira-Ramirez from Mexico in his work on flatness in the first paper of this issue, Han single-handedly reshaped both fields of robust control and disturbance rejection.

In particular, Sira-Ramirez shows how his work of Flatness, GPI Observers, GPI Control and Flat Filters come together under ADRC, in a show of intellectual courage and integrity by "calling things by their proper name", i.e., giving credit wherever it's due. In fact, the author demonstrates that there is a perfect match between the nonlinear system that are flat and the problem of control as formulated in ADRC in terms of the input-output description of a nonlinear plant, where the endogenous perturbation "acts as a matched perturbation input" and is lumped with "the exogenous perturbation" to form what Han called total disturbance. That is, flatness is characteristic of those dynamic systems for which ADRC is readily applicable.

The work on flatness is followed by the work on EMC (embedded model control) by Prof. Enrico Canuto and his collaborators from Italy. The presentation is different this time, though, as the authors show the idea behind EMC as one to reconcile the modern control paradigm based on Kalman's "general theory of control systems" with "the errorbased design paradigm" of Han, for the purpose of resolving the "Han's paradox", i.e., the seemingly unresolvable problem of robustness in model-based designs. The paper begins with Kalman's conception of control theory, the associated problem of uncertainty and robustness described by Doyle and Stein, and the small-gain theorem of Zames, leading to the key insight of "almost-linear plants" of Donati and Vallauri. This is, as authors put it, "the path toward Embedded Model Control" as an engineering solution of the problem of uncertainty and disturbance. A detailed case study was presented to show how EMC provides an effective solution to the quadruple-tank process control problem.

As noted by Canuto et al., Kalman asked, but never directly answered, the right question: "What kind and how much information are needed to achieve a desired type of control?" "If feedback control doesn't require a very accurate knowledge of the plant behavior", said Donati and Vallauri, then "the problem is examined to determine the largest simplification and inaccuracy of the model admissible for a guaranteed feedback control design". These questions, together with Han's, reflect the most foundational issue of modern control theory. Similarly, the issue has also been raised in the context of disturbance rejection in the next paper by Prof. Wen-Hua Chen from U.K.

Chen gives a personal account of how he conceived the Nonlinear Disturbance Observer (NDOB) in the late 1990s as a young postdoctoral researcher: instead of relying on the friction model for a robotic manipulator, he proposed the solution of estimating and cancelling the friction with NDOB, which was named after the work of Disturbance Observer (DOB) by K. Ohishi et al. from the early 1980s. The problem of disturbance in the modern control framework for linear systems was first tackled by Prof. C. D. Johnson's pioneering work in the late 1960s, as noted by Chen, in the form of model-based state observers. NDOB complements the nonlinear control framework by incorporating in it the explicit disturbance estimation and rejection mechanism and, in the case study of robotic manipulators, produces an equivalent nonlinear PID strategy, as shown by Chen, with rigorous mathematical analysis and experimental validations.

From the first three papers we witness in three personal accounts that scholars, across time and space, were driven by the same problem of uncertainty either as a foundational or a practical challenge. The next four "application" papers in this special issue constitute a snapshot and an example of the impact of ADRC, as a general purpose solution in engineering practice. In ADRC engineers finally have a real alternative to PID, as Han predicted ten years ago. 
The second part of this issue starts with the paper "Active disturbance rejection control: Some recent experimental and industrial case studies" by Q. Zheng and Z. Gao, where the ADRC design principle and theoretical justification are briefly overviewed first, followed by examples of practical applications and industry validations. These two authors from the U.S. have firsthand knowledge of transforming the ideas of ADRC into a disruptive technology early on and providing stability proof. The paper is an update from a conference paper from 2012 and it is followed next by three domain-specific application papers, each addresses a sector-wide class of problems.

Prof. S. E. Talole from India opens with an acknowledgement that, yes, feedback has the ability "to reduce the effect of plant uncertainties and external disturbance on the output" but "it takes place passively". An alternative, he notes, is "to obtain an estimate of the lumped or total disturbance" and use it "to nullify its effect". This is of course the central idea of ADRC, conceived by Han in the early days of ADRC development and served as an organizing principle today. Various methods of disturbance observers as well as the associated control strategies, reviewed nicely by Talole, can now be organized and categorized in terms how the disturbance is defined and how the information is obtained. This includes, of course, the methods developed by Prof. Sira-Ramirez, Canuto and colleagues, and Chen, in the first three papers above.

Talole believes that ADRC, as a "desirable strategy", has "immense potential to offer viable solution to real-life aerospace systems", which are highly uncertain but require control design that "can guarantee stability and uniform performance throughout the flight envelope". This is supported by the body of work in the literature in general and the long and sustained work of Talole's group in particular. Talole provides a holistic view on both accounts in this paper.

The next paper by Prof. Jianbo Su's group from China addresses the five core issues in the area of continuous development and evolution of service robots. The problem at hand, hence the motivation, matches almost word for word as that presented by Talole in the previous paper: unmodeled dynamics, external disturbance, strong coupling, nonlinearity, etc. The paper showed some initial results on all five issues but more importantly, it gave a roadmap of what problems to be tackled next and why. Yes, ADRC is a natural fit in obtaining high performance control amid various uncertainties far exceeds what the current toolbox can handle; but it is the prerogative of the domain experts to incorporate the core ideas of ADRC into their control design to form a special and unique solution, not to merely copy the general purpose ADRC as an algorithm.

Finally, the paper by Prof. Lili Dong's group from the U.S. showcases ADRC as an effective solution in two important classes of problems in power systems: load frequency control and voltage regulation. Like any major industry with global significance, there is no shortage of advanced control in power systems. What duly lack are the fundamentally advanced, game-changing, strategies that are easily understood and simply tuned, a need nicely met by ADRC as this paper shows. In addition to simple structure and superior results, the operator only has two parameters to tune, both have clear physical meanings by which common trade-offs are readily made between aggressiveness and robustness.

\section{Outlook}

"With the new outlook and possibilities that ADRC represents, we further believe that control engineering may very well break the hold of classical PID and enter a new era, an era that brings back the spirit of innovations", said Prof. Han in his last paper. That era, in both academic study and industrial technology, may very well be upon us already, if this special issue is of any indication.

ADRC as a field of academic study compels us to reexamine the fundamental concepts, as basic as the very notion of "feedback", "disturbance" and "stability" themselves. In "feedback" we distinguish the kinds of information sent to the controller on which control signal is calculated; in "disturbance" we distinguish the kinds of uncertainty it manifests: within and without, endogenous and exogenous, internal and external; in "stability" we distinguish the kind originated in astronomy and dominated our field to this day, from the kind articulated by H. S. Tsien in 1954: "Stability of any control system means that the design performance of the system will be obtained even with the presence of internal and external disturbances."

Furthermore, in disturbance rejection we distinguish systems that are disturbed from those that are disturbance- 
free, with the goal of reducing the former to the latter. And this is where Han made his biggest mark: the Han Canonical Form (cascade integral, disturbance-free) for dynamic systems, linear or nonlinear, and the concept of total disturbance. While the ensuing transition from PID to ADRC in industrial technology is gradually becoming a reality as he envisioned, the transition from "model theory" to "control theory", as he articulated in 1989, has just begun.

"There is nothing more powerful than the idea whose time has come." - Victor Marie Hugo

Guest editors:

Zhiqiang Gao

Cleveland State University,

Cleveland, Ohio 44115, U.S.A.

Yi Huang

Academy of Mathematics and Systems Science,

Chinese Academy of Sciences,

Beijing, China 